\title{
Las Tecnologías de Información y Comunicación como herramienta para una educación primaria inclusiva
}

Reyes Chávez, Rafael; Prado Rodríguez, Anna Beatriz

Las Tecnologías de Información y Comunicación como herramienta para una educación primaria inclusiva

Revista Educación, vol. 44, núm. 2, 2020

Universidad de Costa Rica, Costa Rica

Disponible en: http://www.redalyc.org/articulo.oa?id=44062184011

DOI: https://doi.org/10.15517/revedu.v44i2.38781

Esta obra está bajo una Licencia Creative Commons Atribución-NoComercial-SinDerivar 3.0 Internacional. 
Revisiones bibliográficas

\section{Las Tecnologías de Información y Comunicación como herramienta para una educación primaria inclusiva}

\section{Information and Communication Technology as Tools for Inclusive Education Systems in Elementary Schools}

Rafael Reyes Chávez

División de Estudios de Posgrado del Departamento de

Ciencias de la Educación de la Universidad Autónoma de

Tlaxcala, México

rafareyesch@hotmail.com

(iD) http://orcid.org/0000-0002-8953-9285

Anna Beatriz Prado Rodríguez

Universidad Autónoma de Tlaxcala, México

anna.b.prado.r1970@gmail.com

(iD) http://orcid.org/0000-0002-2803-1605
DOI: https://doi.org/10.15517/revedu.v44i2.38781

Redalyc: http://www.redalyc.org/articulo.oa?id=44062184011

Recepción: 13 Octubre 2019

Aprobación: 10 Abril 2020

\section{Resumen:}

El presente documento pretende mostrar, a través de una revisión de la literatura, la situación que guarda la educación inclusiva y el papel preponderante que las Tecnologías de la Información y Comunicación (TIC) ofrecen como herramientas de apoyo a la población socialmente vulnerable y desfavorecida. El documento inicia con la conceptualización de inclusión social y educación inclusiva, a continuación se sintetizan los planteamientos señalados por los organismos internacionales, nacionales y locales para la promoción de políticas públicas orientadas a la utilización generalizada de estos recursos para la inclusión de sectores desfavorecidos. Se identifica la formación docente, la disminución de la brecha digital y el diseño de entornos educativos como elementos que detonan o aceleran la inclusión educativa. La metodología consiste en la recolección de 113 documentos publicados en la última década, relacionados específicamente con la educación inclusiva y TIC, además de las políticas de educación inclusiva, de los cuales se han seleccionado aproximadamente la mitad para enriquecer la presente investigación. Finalmente, se asientan las conclusiones pertinentes en la búsqueda de políticas para alcanzar la equidad y justicia que la nueva cultura social requiere, sin embargo, esta es una tarea pendiente que debe ser atendida.

Palabras Clave: Inclusión social, Educación Inclusiva, Tecnologías de Información y Comunicación (TIC), Diseños Universales, Brecha Digital.

\section{ABstract:}

This is a literature review that analyzes the important role of Information and Communication Technologies (ICTs) in inclusive education and how it functions as support tools for underprivileged and vulnerable population groups. Conceptual approaches of social inclusion and inclusive education proposed by local, national and international organizations are initially summarized. Such approaches are used to promote public policies for the overall use of these resources to serve the underprivileged. Teacher education, reduction of the digital gap and design of educational surroundings are key elements that trigger or accelerate educational inclusion. The methodology for this study consisted of a review of 113 articles published during the last decade that specifically cover inclusive education and ICTs. A selection of approximately half of inclusive educational policies was also analyzed to enrich this article. The conclusions drawn emphasize that new social cultural factors require policies that will help attain equality and justice, a pending item that must be addressed.

KEYWORDS: Social Inclusion, Inclusive Education, Information and Communication Technology (ICT), Universal Designs, Digital Gap. 


\section{INTRODUCCIÓN}

La inclusión educativa a nivel internacional se ha convertido en un foco central de debate de las políticas públicas en educación, sumado a ello cada vez es más reconocido el papel preponderante que las TIC tienen en todos los aspectos de la vida humana, y la educación no es una excepción, en esa misma línea el texto pretende aportar elementos que favorezcan la inclusión de las TIC como herramientas de apoyo a proceso de inclusión a la población socialmente vulnerable y desfavorecida.

Se puede enunciar el fenómeno de las TIC y su impacto en el ámbito educativo bajo dos posturas, la primera hace mención a su incorporación como elemento que promueve la desigualdad, pues no todos pueden acceder a las herramientas y recursos tecnológicos; por lo tanto, dividen a las personas entre quienes tienen acceso, habilidades para su manejo y utilización, de aquellas que están marginadas de estos recursos, es decir, ahora se habla de las diferencias ya no solo de espacios de desarrollo, sino de las fronteras que se miden en términos de la alfabetización digital (Ortoll, 2007). Además, para Ortoll (2007) la inclusión digital admite comprender las oportunidades que ofrecidas, como acercar (beneficios) el manejo de las TIC en una comunidad debe reflejar una relación con la necesidad en particular presentada al colectivo (cohesión social).

Por otro lado, Caridad, Calzada, Jorge y Ayuso, (2011) al igual que ubican a las TIC como un medio que puede representar para las personas socialmente desfavorecidas una importante oportunidad de inclusión (acceso a información, formación, trabajo, salud, comunicación y expresión); de tal forma que, la inclusión social se vincula directamente con el uso de las TIC mediada por la sociedad del conocimiento, como lo refiere Souter (2010):

Las nuevas tecnologías han generado nuevas oportunidades para la creación, preservación, difusión y uso de la información, pero es la actividad humana la que permite a la información ser transformada en conocimiento, y al conocimiento mismo añadir valor a la experiencia y desarrollo humano (...) (p. 12).

Hay que considerar que, la sola presencia de las TIC no asegura el proceso de inclusión; por el contrario, su acertado uso y manejo sirven como herramientas que favorecen la inclusión social. En el ámbito educativo las TIC ofrecen una variedad de oportunidades para el desarrollo de procedimientos promotores de una educación inclusiva, colaboradoras durante el proceso de enseñanza-aprendizaje en los diferentes niveles educativos, dentro y fuera del aula. La presente revisión bibliográfica pretende reconocer a las TIC como herramientas facilitadoras de los sistemas de educación inclusiva a nivel primaria, exponer algunos de los recursos que ofrecen dichos sistemas, de igual forma, propuestas de políticas de inclusión en la educación.

Para Cabero y Córdoba (2009, citado por Balladares, 2019), son necesarias tres condiciones para llevar a cabo una inclusión digital educativa:

Primero debe haber presencia de las TIC; después, acceso y uso de la tecnología; y, por último, generar un proceso de alfabetización digital para conocer los lenguajes y sistemas simbólicos de una sociedad de la información y del conocimiento (p.199).

\section{INCLUSIÓN SOCIAL}

El concepto de inclusión social presenta una gran amplitud de definiciones por diferentes organismos internacionales; como la Organización de Naciones Unidas a través Organización de las Naciones Unidas para la Educación, la Ciencia y la Cultura [Unesco], Organización de Estados Americanos [OEA], Comisión Económica para América Latina y el Caribe [CEPAL], la Unión Europea [EU], entre otros (Cabero, 2005), estableciendo diversas políticas entre ellas las de inclusión social y son aceptadas por sus países miembros quienes firman los acuerdos. Dichas políticas se ajustan a las normas de cada país, de acuerdo con su Constitución Política y México no es la excepción. Sin embargo, la definición sobre inclusión social sugerida por la EU es la considerada adecuada para el presente trabajo, siendo esta un proceso el cual garantiza aquellas 
personas en desventaja de obtener las oportunidades y recursos necesarios para participar plenamente en la vida económica, social y cultural permitiéndoles el goce de un estilo de vida considerado normal en la sociedad que vive, así como el acceso a sus derechos fundamentales (Caridad et al. 2011).

En un texto reciente, Watts y Lee (2017) mencionan que para el Banco Mundial (2011) el concepto de inclusión social implica un proceso de empoderamiento para las personas o grupos facilitando su participación dentro de la sociedad, de tal forma que puedan aprovechar las oportunidades; mientras que, la CEPAL la considera como un proceso para lograr la igualdad permitiendo sortear sus limitantes en la productividad, educación, empleo, segmentación laboral e informalidad.

Además, Watts y Lee (2017) aseguran que la educación desea "proporcionar oportunidades de crecimiento profesional y personal a través de experiencias significativas que conduzcan al desarrollo de habilidades, conocimientos, aptitudes y hábitos útiles para vivir y servir a la sociedad" (p. 93), advirtiendo la importancia de la inclusión social como la igualdad de oportunidades con la aceptación general de sus miembros, considerándola una estrategia fundamental para superar las desigualdades (personales, culturales y económicas) que son consecuencia de discapacidad.

Para Caridad et al. (2011) el concepto de inclusión social se origina en oposición positiva al de exclusión social, en el mismo sentido Blanco (2014) y Cabero (2005) mencionan que la exclusión y marginación en el ámbito educativo son el producto de diversos factores dentro de sus sistemas (personales, sociales, culturales y económicos). En adición con lo anterior, Lázaro, Estebanell, y Tedesco (2015) asignan un valor de justicia social a la inclusión digital, pues mejora la calidad de vida mediante la accesibilidad a los servicios digitales, alfabetización digital, uso responsable de las TIC, así como el acceso a la formación y al mercado laboral.

En resumen, se identifica que la sociedad del siglo XXI exige la observación de la estrecha relación entre inclusión social y digital, puesto que al favorecer la inclusión digital en el colectivo, logra mejorar su calidad de vida, asegurando con certeza el proceso de inclusión social que actualmente nuestra sociedad demanda; inclusive, los diversos sistemas educativos desarrollados por los diferentes países se ven inmersos dentro de esta dinámica para promover la inclusión social a través de la digital, estableciendo el nuevo reto de la educación inclusiva.

\section{EDUCACIÓN INCLUSIVA}

El alumnado con necesidades especiales ha tenido diversos tratamientos a lo largo de la historia de la educación, en un primer momento, hasta los años setenta del siglo XX fueron motivo de segregación y aislamiento en el sistema educativo. Como alternativa a estos modelos segregados de currículos y escuelas para necesidades especiales surge, en la década de 1980, el concepto de integración con el objetivo de que el estudiantado considerado con necesidades especiales ingresara en las escuelas regulares; así, se llevó a cabo el cierre de escuelas especiales para ingresar al alumnado en escuelas comunes, mas no fue suficiente para el reconocimiento de la diversidad de expectativas y necesidades de los educandos (Samaniego, Laitamo, Valerio, y Francisco, 2012). Todo esto dio impulso a una tercera etapa en la década de los noventa, la cual moviliza un cambio notable del alcance, objetivos, contenido e implicaciones de lo que en la actualidad denominamos educación inclusiva.

Cabe señalar que, Cabero y Valencia (2019) diferencian los conceptos de inclusión e integración, el primero se centra en cada estudiante, mientras que el segundo, en el aula; es decir, la inclusión se enfoca en un modelo individual donde las dificultades y limitantes comprometen al alumnado a superarlas, además de ser este quien se adapte al contexto educativo inmerso. Por tanto, la educación inclusiva persigue mejorar la calidad educativa de sus miembros (estudiantes, profesores, familias, entre otros) para promover una educación para todos, concebida como un derecho humano de búsqueda de calidad y sentido social (Cabero y Valencia, 2019; Cabero y Ruiz, 2018); aclarando que, la educación inclusiva no es sinónimo de integración escolar, a pesar de su estructuración impuesta por los sistemas educativos (Cabero y Córdoba, 2009). 
Generalmente el concepto de educación inclusiva se comprende como aquella exclusiva de personas con alguna imposibilidad física, mental o psicológica, quienes requieren cuidados especiales dentro de un ambiente particular para facilitar su aprendizaje; es decir, esta educación se asocia con personas que presentan una discapacidad biológica o psicológica y representa una problemática social (Rodríguez y Arroyo, 2014; Cabero y Fernández, 2014).

En su página web, la Organización Mundial de la Salud (OMS) (2019) define la discapacidad como "un término general que abarca las deficiencias, las limitaciones de la actividad y las restricciones de la participación” (párr. 1); entendiendo por deficiencias aquellos problemas que afectan estructural y funcionalmente el cuerpo, por limitaciones las dificultades para ejecutar tareas o actividades y restricciones esos obstáculos que se presentan en la vida cotidiana (Carrillo, y Molina, 2016). No obstante, los cambios señalados anteriormente no han logrado homogeneidad en el manejo lingüístico y aún persisten vocablos peyorativos para referirse a las personas que presentan alguna discapacidad.

Además, la UNESCO (2014) establece que los países miembros tienen que garantizar “(...) un sistema educativo inclusivo en todos los niveles, una educación gratuita y obligatoria en igualdad de condiciones para acceder a la educación, realizar trayectorias educativas satisfactorias y tener éxito en su aprendizaje” (p.71); determinando que la educación de calidad es un derecho para las personas con discapacidad o aptitudes sobresalientes (Gallegos y López, 2018).

Por consiguiente, el objetivo de la educación inclusiva, según Cabero y Fernández (2014), es lograr la equidad e igualdad de los derechos entre todas las personas, es decir, con el alumnado en situación vulnerable tiende a ser excluido del sistema educativo (Levens, 2011); tales como, personas menores con alguna discapacidad, quienes viven en pobreza y marginación, quienes pertenecen a grupos étnicos y hablan lenguas minoritarias, quienes viven en zonas remotas y las personas marginadas como mujeres, tercera edad, en desempleo, analfabetismo e inmigrantes. Además, con la inclusión se pretende alcanzar "la transformación cultural, la organización educativa y la práctica escolar” (Cabero y Fernández, 2014, p. 38).

Aunado a lo anterior, se destaca que durante las VIII Jornadas de Cooperación Educativas con Iberoamérica (2011), organizadas por la UNESCO, se obtuvieron conclusiones, enfatizando la siguiente:

Los sistemas educativos inclusivos favorecen la participación, el aprendizaje y el máximo desarrollo de las potencialidades de cada persona, son el medio por excelencia para vivir juntos en la diversidad, valorando las múltiples identidades y contribuyen a la construcción de sociedades más justas y cohesionadas (Unesco, 2014, p. 71).

De acuerdo con Blanco (2014) la educación inclusiva debe responder a la diversidad del alumnado, aumentando su participación cultural, el currículo y las comunidades de las escuelas, de tal manera que, se disminuya la exclusión en y desde la educación. Ahora bien, para lograrla el sistema educativo debe contar con un currículo flexible, promover mecanismos de apoyo y democráticos que garanticen la atención, así como la participación de sus integrantes, refrendando la reflexión y descentralización de la escuela (Unesco, 2009). Por eso, el Consejo Nacional para el Desarrollo y la Inclusión de las Personas con Discapacidad (CONADIS) (2015) aclara que el concepto de inclusión no es propio del sistema educativo, por el contrario, es la perspectiva de una sociedad incluyente que respeta el valor y lugar que ocupa cada uno de sus miembros. Es decir, el CONADIS (2015) considera la educación inclusiva como:

Un principio rector destinado a alcanzar niveles deseables de integración escolar de todos los estudiantes supone la formulación y aplicación de estrategias de aprendizaje que den respuesta a la diversidad del alumnado, generando las bases de una educación con las mismas oportunidades para los niños, niñas y adolescentes con discapacidad (párr. 8).

En síntesis, en México, el CONADIS (2015) refiere la existencia de normas con las que se sustenta el desarrollo de educación inclusiva en nuestro país, siendo las más destacadas las que a continuación se enlistan:

a) Constitución Política con el artículo $3^{\circ}$ el cual establece el derecho a la educación. 
b) Convención sobre los Derechos de las Personas con Discapacidad en su artículo $24^{\circ}$ que menciona la educación inclusiva en todos los niveles y a lo largo de la vida.

c) Ley General para la Inclusión de las Personas con Discapacidad en el artículo $12^{\circ}$ donde la Secretaría de Educación Pública [SEP] es la encargada de garantizar esta educación mediante diferentes acciones.

d) Programa Nacional para el Desarrollo y la Inclusión de las Personas con Discapacidad 2014-2018 (PNDIPD) en objetivo 4, fortaleciendo la participación por medio de estrategias y líneas de acción.

e) Programa Sectorial de la SEP proponiendo atender las necesidades educativas de las personas con discapacidad.

f) Revisión del modelo educativo para ejecutar cambios metodológicos, curriculares, de actitud y compromiso los cuales favorezcan la enseñanza-aprendizaje.

A continuación, Velasco (2017) desarrolla una breve reseña histórica sobre las normas que fundamentan la inclusión de personas discapacitadas en México, destacando que a partir del 2005 se crea la Ley General de las Personas con Discapacidad, la cual contaba con antecedentes de derechos e inclusión; posteriormente, se retoma el concepto de inclusión en la Convención sobre los Derechos de las Personas con Discapacidad y su Protocolo Facultativo, el cual entra en vigor en mayo del 2008; al año siguiente, el Programa Nacional para el Desarrollo de las Personas con Discapacidad 2009-2012, y el referente más reciente, el Programa Nacional para el Desarrollo y la Inclusión de las Personas con Discapacidad 2014-2018.

Para Cabero, Fernández y Córdoba (2016) las TIC son instrumentos significativos que favorecen la inclusión de las personas con deficiencias de tipo cognitivas, sensoriales o motriz, otorgando ayuda para superar sus limitaciones, beneficiando la independencia y autonomía, la adaptación a las necesidades y demandas personales, la comunicación sincrónica y asincrónica entre pares y docentes, el respaldo de un modelo de comunicación y formación multisensorial, evitando la marginación y la brecha digital, entre otras cosas. Actualmente, se observa el esfuerzo por incrementar la presencia de las TIC, además de potenciar la atención a las personas con discapacidad y en situaciones de exclusión; para ello, se ha intervenido en la protección y promoción de los derechos e inclusión de las personas con discapacidad (Cabero y Valencia, 2019).

Al advertir la implicación que presenta el concepto de inclusión en la educación, tal como lo define Cabero, Fernández y Córdoba (2016), despierta en los especialistas una visión más reflexiva y activa para atender esta nueva meta que debe ser alcanzada, identificando que la inclusión necesariamente es para los más vulnerables. Donde una de las propuestas es el uso asertivo de las nuevas tecnologías educativas, permitiendo superar sus limitaciones o deficiencias además del desarrollo de políticas y normas que protejan los derechos de las personas desamparadas.

\section{NuEVAS TECNOLOGÍAS EN EDUCACIÓN INCLUSIVA}

Las TIC juegan un papel muy importante en la sociedad del siglo XXI, también conocida como sociedad del conocimiento. Castells (2000) refiere que las nuevas tecnologías de la información no pueden ser vistas como una herramienta para ser aplicadas, sino como procesos que se deben desarrollar.

Cada vez más autores aseguran que el uso de las tecnologías digitales puede ayudar al desarrollo de la educación inclusiva, permitiendo de manera efectiva la democratización del conocimiento y la participación de sus actores (Cabero y Fernández, 2014; Caridad et al., 2011; Gobierno de la República, 2013). También apoyan firmemente lo anterior Lázaro et al. (2015) donde reconocen que "las políticas educativas inclusivas deberían implementarse, además de asociarse a los principios y valores siguientes: acceso y calidad, igualdad y justicia social, democracia y participación, y equilibrio entre comunidad y diversidad" (p. 5). 
Además, estos mismos autores afirman que el internet funciona como un agente catalizador para la educación inclusiva, por su factor de acceso generalizado a la tecnología que facilita la inclusión y la cohesión social gracias a la democratización del conocimiento; para ello, Lázaro et al. (2015) proponen tres líneas de acción:

a) La disponibilidad de equipamientos digitales públicos y gratuitos, como las redes de bibliotecas, telecentros o zonas de libre acceso a la red vía WiFi, son opciones que favorecen el acceso universal.

b) La estrategia del diseño para todos tiene un papel clave en la creación de sociedades inclusivas y, por tanto, debería incorporarse a todos los niveles del proceso de diseño de bienes y servicios.

c) Las TIC en educación deberían adaptarse a las necesidades de todos los estudiantes, particularmente a aquellos con necesidades educativas especiales (p.9).

Complementariamente, Samaniego et al. (2012) citan que los países latinoamericanos más avanzados en la implementación de la segunda generación de políticas de TIC son Uruguay y Chile; los demás se encuentran en la primera etapa, siendo Argentina, Colombia, Perú y Venezuela quienes se ubican en la fase de ejecución de la primera generación de las agendas digitales; los que están en la fase de formulación son Bolivia, Brasil, Ecuador; por último, Paraguay se encuentra en la de origen. Ejemplo de ello son Chile con la política Empoderamiento Digital de Personas con Discapacidades, Costa Rica y su programatecno@prender, Colombia con el programa Democratización de la Innovación en las Américas y en México, el Programa de Inclusión Digital (Organización para la Cooperación y Desarrollo Económicos [OCDE], 2018) bajo los objetivos de conectividad, contenidos y servicios digitales, e inclusión digital (Palacios, Flores y García, 2013). Aunado a ello, Cabero (2010) destaca que dentro del informe Horizon para Latinoamérica se contemplan los siguientes retos en un intento por incorporar las TIC en las instituciones educativas:

a) Formación docente en el uso de medios digitales en los procesos de enseñanza y aprendizaje.

b) Gestión del cambio integral en la educación superior desde un enfoque sistémico y transformador, que contribuya al crecimiento económico, el desarrollo humano y la cohesión social.

c) Alfabetización digital como aptitud esencial de la profesión docente.

d) Formación de los estudiantes en el uso de los nuevos medios y lenguajes de comunicación audiovisual.

e) Uso de la tecnología para un tratamiento adecuado de la información y la construcción de conocimiento.

f) Adaptación de las prácticas docentes a los requerimientos de la sociedad digital y del conocimiento.

g) Integración y uso de la tecnología al servicio de la enseñanza y del aprendizaje (p. 51)

Crear políticas para la incorporación de TIC en las escuelas no necesariamente favorece por sí mismo el desarrollo de la educación inclusiva, Cabero y Fernández (2014) sugieren además reflexionar sobre su diseño, así como las competencias que debe tener el profesorado para su utilización, esto implica que debe poseer competencias digitales en uso de dichas tecnologías, las cuales evidencien el proceso de enseñanzaaprendizaje.

De acuerdo con lo anterior, otro de los factores claves para la inclusión educativa recae en la formación permanente del claustro, según Lázaro et al. (2015) proponen las siguientes líneas de acción:

a) Contar con programas de formación inicial que tengan continuidad en la formación permanente, tanto en el diseño instruccional como en la aplicación de los criterios de accesibilidad mediante las técnicas adecuadas para las tecnologías que van emergiendo.

b) Definir políticas de apoyo a las redes y repositorios de los recursos existentes, con el fin de incrementar su alcance y atractivo para los profesores, así\# como para garantizar su adecuación a los requisitos de accesibilidad. 
c) Los principios del diseño para todos, las estrategias de diseño instruccional y, en particular, las técnicas de accesibilidad digital deben formar parte esencial de la formación del profesorado en todos sus niveles.

d) Al igual que la formación permanente es imprescindible, también lo es la evaluación permanente, puesto que le proporciona alicientes al profesorado para que se anime a someterse a ella y a avanzar en la aplicación y el reconocimiento de buenas prácticas de educación inclusiva. (p.11)

García y López (2012) describen las funciones de integración de las TIC dentro del aula (p. 280):

1. Acceso: Aprender a utilizar correctamente la tecnología.

2. Adopción: Apoyar a una forma tradicional de enseñar y aprender.

3. Adaptación: Integración en formas tradicionales de clase.

4. Apropiación: Uso colaborativo, proyectos y situaciones necesarias

5. Innovación: Descubre nuevos usos de la tecnología y combinan las diferentes modalidades.

Bajo esa misma perspectiva, Lázaro et al. (2015) consideran que si se asegura la interacción de las funciones de las TIC con los modelos de atención a la diversidad, ciertamente se pueden integrar las funciones de acceso, adopción y adaptación, es decir, aquellas que no cuestionan el statu quo escolar; mientras que los usos inclusivos desarrollarían tareas de apropiación e innovación, o sea, las que permiten la trasformación escolar.

La definición que ofrece la OCDE sobre las TIC son aquellos dispositivos que capturan, transmiten y despliegan datos e información electrónica que apoyan el crecimiento y desarrollo económico de la industria manufacturera y de servicios (Baelo y Cantón, 2009). Sin embargo, no pueden ser consideradas la panacea en la innovación educativa por su sola aplicación, éstas deben ser integradas a la cultura de cambio e incorporadas al currículo educativo (Gobierno de la República, 2013), así como la formación y desarrollo docente, el diseño instruccional, los recursos educativos digitales, iniciativas estratégicas, equipamiento, conectividad, monitoreo y evaluación (SEP, 2016; Vaillant, 2013).

Ahora bien, de acuerdo con Caridad et al. (2011) una persona puede ser excluida digitalmente al privársele de la oportunidades y derechos de acceder al uso de las TIC o en su defecto cuando no tiene capacidad de disfrutarlas, por consiguiente, la apropiación efectiva de las TIC es una condición necesaria para lograr la participación activa en la sociedad; estas podrían ser un medio de oportunidad de mejora e inclusión para una persona desfavorecida socialmente (acceso a información, formación, trabajo, salud, comunicación y expresión) (OCDE, 2017). Por ello las organizaciones mundiales, como la UNESCO y la OEA, pretenden destacar la dimensión humana de la información y comunicación para alcanzar la democratización, inclusión y justicia social (Secretaría de Acceso y Equidad de Derechos [SADYE], 2016) dentro de la sociedad del conocimiento.

El término de alfabetización informacional, mencionado por Caridad et al. (2011) es referente de las competencias transversales relacionadas con el acceso, manejo y uso de la información, siendo una de las herramientas disponibles para aminorar la brecha digital entre los individuos y no solo vincularla con la inclusión digital, también con las diversas iniciativas de política para el avance de la sociedad del conocimiento, además de la alfabetización informacional se identifican la tecnológica, organizativa, mediática, visual y cultural (Watkins, 2013).

La tecnología educativa es el apoyo que ofrece la tecnología al servicio de la educación, esto puede permitir el desarrollo de la educación inclusiva favoreciendo la educación de calidad, eliminando las barreras que impiden el acceso al acto educativo y la cultura (Cabero y Fernández, 2014); a través de la flexibilización a nivel temporal-espacial para la interacción y recepción de la información y/o del conocimiento, facilitando la búsqueda de nuevas perspectivas conforme a las variables y dimensiones del acto educativo (Cabero, 2010). 
Las TIC orientadas al sector vulnerable pueden ser una gran oportunidad educativa para alcanzar la inclusión en la sociedad del conocimiento a través de la creación de ambientes que faciliten aprendizajes (Escobar, Restrepo y Castrillón, 2016), disminuyendo el déficit social, escolar, económico y cultural.

Altamirano y Lera (2017) confirman que las tecnologías de la información suponen un impacto democratizador además de incrementar los logros de aprendizaje de los estudiantes gracias a:

a) Los cambios en los procesos y estrategias didácticas implementadas por los docentes.

b) La promoción de experiencias de aprendizaje más creativas y diversas

c) La posibilidad de propiciar un aprendizaje independiente y permanente de acuerdo a las necesidades de los sujetos (p. 424)

Actualmente, a aplicación de las TIC en los procesos formativos de educación se complementan (Cabero y Fernández, 2014). Las TIC son entonces percibidas como facilitadores y trasmisores de información, así como de recursos educativos para estudiantes; las TAC (Tecnología Aprendizaje y Conocimiento) utilizados como facilitadores del aprendizaje y conocimiento entre el cuerpo docente y el alumnado, además permite la comunicación del aprendizaje; por último, las TEP (Tecnología para el Empoderamiento y la Participación) son instrumentos de participación y colaboración de cada docente con el estudiantado, percibiendo la tecnología como mediador en la construcción del conocimiento y la interacción social (Cabero, 2016; Cabero y Ruiz, 2018). La Figura 1 muestra el proceso de desarrollo que tiene la tecnología del aprendizaje.

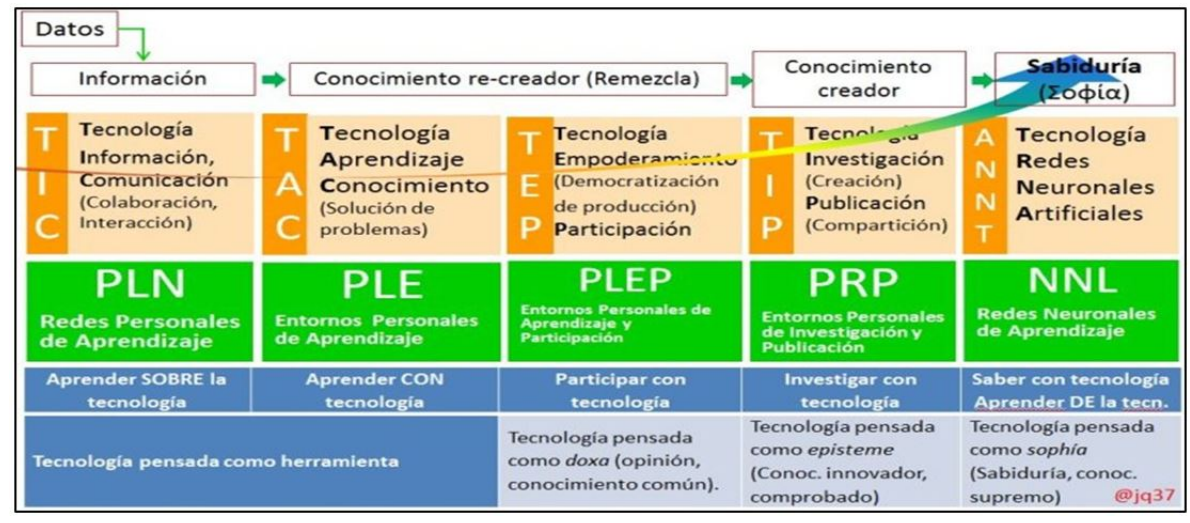

FIGURA 1

Tecnologías para aprender

Fuente: Prioretti (2016).

Con relación a las discapacidades y TIC, Cabero (2008) plantea su acercamiento bajo dos vertientes, una es aprovechar las TIC para apoyar a personas con diferentes tipos de discapacidades en una mejor incorporación con la sociedad, relación con el medio ambiente, además de comunicarse e interaccionar con el resto de ciudadanía, y la otra prestar especial atención en su diseño e incorporación para la formación y el mundo laboral; convirtiéndose en instrumento de inclusión social; exponiendo sus ventajas y desventajas en la Tabla 1 . 
TABLA 1

Enseñanza a través de multimedia y TIC

\begin{tabular}{|c|c|}
\hline Ventajas & Desventajas \\
\hline Alto poder motivante. & $\begin{array}{l}\text { Su utilización depende del tipo de } \\
\text { discapacidad al que nos estemos } \\
\text { refiriendo: visual, auditiva, motriz, } \\
\text { cognitiva, etc. }\end{array}$ \\
\hline $\begin{array}{l}\text { Creación de entorno rico con la } \\
\text { incorporación de diferentes sistemas } \\
\text { simbólicos, que facilitan la creación de } \\
\text { entornos dinámicos y atractivos. }\end{array}$ & $\begin{array}{l}\text { Integración depende del tipo de } \\
\text { discapacidad y su grado }\end{array}$ \\
\hline $\begin{array}{l}\text { Flexibilización de los entornos de } \\
\text { comunicación. }\end{array}$ & $\begin{array}{l}\text { Utilización conforme al hardware } \\
\text { (componente físico de los ordenadores: } \\
\text { teclados, impresoras, monitores, etc.) y el } \\
\text { software (componente lógico: programas } \\
\text { informáticos, navegadores, etc.). }\end{array}$ \\
\hline $\begin{array}{l}\text { Repetición, de los fragmentos de } \\
\text { información y ejercicios. }\end{array}$ & $\begin{array}{l}\text { Adaptación de los medios } \\
\text { convencionales, con los específicos }\end{array}$ \\
\hline $\begin{array}{l}\text { Adaptación a las características } \\
\text { individuales de los sujetos }\end{array}$ & $\begin{array}{l}\text { La investigación y análisis de diferentes } \\
\text { profesionales (pedagogos, ingenieros, } \\
\text { psicólogos, diseñadores, etc.). }\end{array}$ \\
\hline
\end{tabular}

Fuente: Elaboración propia con datos de Cabero (2008)

Cabero (2015) habla de un concepto concebido como la desigualdad de acceso que se presenta en los individuos e instituciones por las tecnologías a través de las cuales se produce y distribuye la información al igual que el conocimiento, importantes en la sociedad actual. Para él, no solo se limita al acceso de dichas tecnologías, sino a las diferentes competencias y capacidades que permiten su utilidad específica, como su calidad, contexto y diversos niveles de empleo.

La brecha digital al ser amplia, muestra mayor exclusión hacia las personas que no pueden acceder a las TIC por motivos económicos, sociales o educativos; de ahí que, la inclusión presupone disminución de la pobreza $y$, consecuentemente, a minora dicha brecha. Es necesario destacar que la brecha digital se debe abordar bajo un enfoque global, como un derecho concebido por los individuos a no ser excluidos de la nueva sociedad del siglo XXI, para mejorar la calidad de vida (Watkins, 2013). Presentando una relación directa con el índice de inclusión digital y el aumento en la mejora de la accesibilidad a los servicios digitales, la alfabetización digital, la igualdad, el uso responsable de la tecnología, así como el acceso a la formación y al mercado laboral (Lázaro et al., 2015). En la Tabla 2 se especifican los tipos de brecha digital y sus características.

TABLA 2

Tipos y características de brecha digital

\begin{tabular}{|c|c|c|}
\hline \multicolumn{3}{|l|}{ Brecha Digital } \\
\hline Digital & Análoga & Axiológica \\
\hline $\begin{array}{l}\text { Conectividad, } \\
\text { infraestructura y } \\
\text { dispositivo de } \\
\text { acceso. }\end{array}$ & $\begin{array}{l}\text { Educación, } \\
\text { trabajo e ingreso. }\end{array}$ & $\begin{array}{l}\text { Creencias, valores, } \\
\text { actitudes, } \\
\text { expectativas y } \\
\text { deseos. }\end{array}$ \\
\hline
\end{tabular}

Fuente: Elaboración propia con datos de Thomas, Juárez y Picabea (2015).

$\mathrm{Al}$ reconocer a las tecnologías digitales como herramientas que apoyan la educación inclusiva, su responsabilidad del manejo y uso dentro del aula por parte de las y los docentes provoca en ellos resistencia en el desempeño laboral; puesto que consideran que a mayor manejo de TIC, más presión laboral, esto lo establece Sánchez (2014). Por lo tanto, Sánchez (2014) expone la perspectiva de transición del paradigma 
denominado deficitario al emergente competencial, es decir aquel relacionado médicamente y poco a poco se elimina su dependencia; de tal forma que se permite clasificar a las TIC en exclusiva, de apoyo e invisibles. Lo anterior se expresa en la Figura 2, en la trascendencia del paradigma deficitario al competencial.

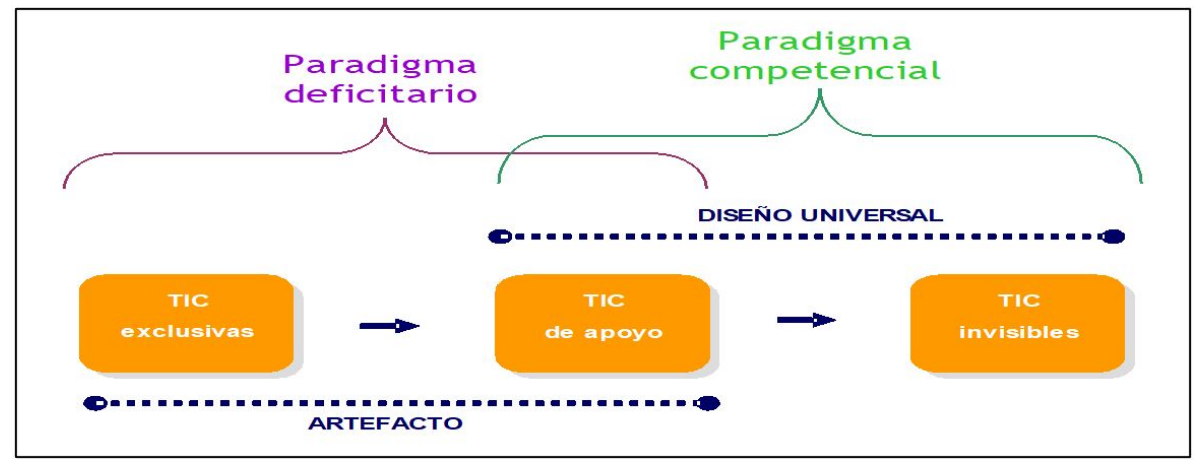

FIGURA 2

Las cosmologías se entrelazan e influyen Fuente: a partir de Sánchez (2014)

En esta figura, las TIC exclusivas no son para todas las personas, solo son eficientes para aquellas que poseen una discapacidad específica, son de uso fácil y cumplen su objetivo de manera limitada, ya que con el tiempo estas no crecen con la persona. Mientras que las TIC de apoyo o Assistive Technology son conocidas como rampas digitales, es decir los programas de software con Diseño Universal (DU) que son útiles para todos los individuos, sin importar su discapacidad; estas son diseñadas como un producto estándar para el usuario. Por último, las TIC invisibles son aquellas que desaparecen al momento de usarlas; Donald Norman (1998, citado por Sánchez, 2014), refirió "que la tecnología este\# detrás y no delante de las tareas" (p.42), en otras palabras, que el espacio entre el usuario y la TIC se reduzca hasta llegar a ser invisible.

Ejemplo de este tipo de TIC son las interfaces directas (biónicas), rampas digitales o Assistive Technology, software gratuito y tecnología de bajo costo para la inclusión. Si se habla de la TIC invisible o de rampa digital se infiere que trata de un DU o diseño para todos, el cual se caracteriza por evitar los productos específicos para personas con una determinada discapacidad; este producto (software y/o hardware) se vislumbra como un concepto ecológico (Sánchez, 2014).

$\mathrm{Al}$ comprender lo que es un DU, será más fácil definir el término de Diseño Universal de Aprendizaje (DUA) pues son TIC que deben ser diseñadas, de tal manera que permitan la inclusión y no aumenten la discapacidad (Cabero y Fernández, 2014). Sobre este rubro, Watkins (2013) refiere la necesidad de aplicar principios inclusivos por diseño adaptados a las necesidades de estudiantes especiales, reduciendo las desigualdades en la educación.

Cabero y Fernández (2014) recomiendan una serie de elementos o principios en los cuales debe estar fundamentado el DUA, y son:

a) Igualdad de uso. El diseño debe ser fácil de usar y adecuado para todas las personas independientemente de sus capacidades y habilidades.

b) Flexibilidad. El diseño debe poder adecuarse a un amplio rango de preferencias y habilidades individuales.

c) Simple e intuitivo. El diseño debe ser fácil de entender independientemente de la experiencia, los conocimientos, las habilidades o el nivel de concentración del usuario.

d) Información fácil de percibir. El diseño debe ser capaz de intercambiar información con el usuario, independientemente de las condiciones ambientales o las capacidades sensoriales del mismo.

e) Tolerante a los errores. El diseño debe minimizar las acciones accidentales o fortuitas que pueden tener consecuencias fatales o no deseadas. 
f) Escaso esfuerzo físico. El diseño debe poder usarse eficazmente y con el mínimo esfuerzo posible.

g) Dimensiones apropiadas. Los tamaños y espacios deben ser apropiados para el alcance, manipulación y uso por parte del usuario, independientemente de su tamaño, posición o movilidad (p. 40)

El Diseño Universal de Entornos Educativos (DUEE) contribuye en todos, tanto estudiantes con discapacidad como el personal docente y administrativo, para contar con planteles más seguros y amables para todos, garantiza la participación y sobre todo asegura el aprendizaje y el desarrollo de las competencias para la vida del alumnado con discapacidad o sin ella.

Además, Zappalá, Köppel, y Suchodolski, (2011) admiten que para las personas con dificultades de aprendizaje y participación, el DU brinda un ambiente de cercanía tecnológica, el cual cumple con las características elementales invisibilidad, ubicuidad y adaptabilidad; por lo tanto, la Figura 3 exhibe su propuesta de un esquema genérico sobre las características que conforman un DU.

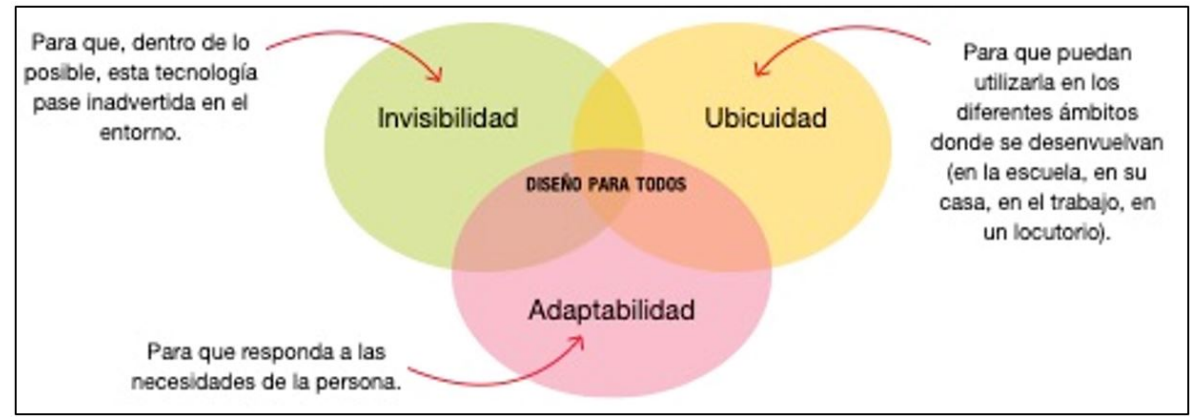

FIGURA 3.

Diseño universal genérico

Fuente: Zappalá et al. (2011).

Cualquier DUA deberá impulsar el trabajo con las TIC a lo largo del proceso enseñanza-aprendizaje a través de los múltiples medios de presentación, donde estas funcionan como estimulantes de la percepción multisensorial; varios medios de expresión, el particular estilo de cada estudiante el cual es reflejado a lo largo de este proceso; además, diversos medios de motivación, las TIC son agentes activos en el aula y permiten el aprendizaje (Cabero y Fernández, 2014). Incluso los formatos accesibles y recursos de acceso libre son herramientas tecnológicas que aseguran la democratización de la información y el conocimiento (Escobar, Restrepo y Castrillón, 2016); Samaniego et al. (2012) enlistan los recursos libres y especifican sus características:

a) Las plataformas tecnológicas de uso más frecuente son: televisión, radio, teléfonos móviles e Internet

b) La radio, la televisión y otras formas tradicionales para difundir información, así\# como los medios de comunicación, no son accesibles para personas con discapacidad.

c) Son muy escasos los programas con 'close caption' y audio-descripción, más como excepción que como práctica continua.

d) Muchas páginas web, no son accesibles a pesar de que la normativa así\# lo estipula (p.19).

Se reconocen en las tecnologías su apoyo en la educación inclusiva, en particular educación especial, confirmando que la computadora es una herramienta clave para trabajar con menores discapacitados (Rodríguez y Arroyo, 2014). Por eso, Rodríguez y Arroyo (2014) sustentan los siguientes beneficios del uso de la computadora:

a) Ahorra tiempo y esfuerzo.

b) Introduce una dinámica visual muy importante, sobre todo para niños/as con problemas de lenguaje.

c) Incrementa la motivación y refuerza la atención. 
d) Prepara al niño/a para el aprendizaje permanente y autónomo.

e) Contribuye a las capacidades de autoaprendizaje estimulando la creatividad.

f) Ofrece la posibilidad de una evaluación dinámica continua y objetiva.

g) Contribuye a la adquisición de capacidades básicas como son la lectoescritura, la expresión, el cálculo, el pensamiento lógico, la resolución de problemas, etc. (p.112)

\section{Propuestas PARA UNA POLÍTICA DE INCLUSIÓN EDUCATIVA}

Actualmente, se vive una nueva etapa denominada nueva revolución industrial, consistente en la penetración que tiene la tecnología en nuestra sociedad y provocando cambios significativos en diversos ámbitos. En dicha revolución se acentúa el rol que desempeña la innovación en la tecnología, economía y el sector industrial, mediante la automatización progresiva del trabajo sustentado en el desarrollo tecnológico y la difusión del conocimiento, este último viaja en forma de datos por la red generando nuevas formas de conocimiento; al ser condicionado y modificado nuestro entorno se producen consecuencias que provocan desigualdad y exclusión (Lázaro et al., 2015).

Asimismo, Cabero y Valencia (2019) aseguran que las transformaciones requeridas en la educación deben disminuir la brecha digital y favorecer la inclusión mediante las TIC, siendo primordial que el estudiantado tengan un mínimo de competencias instrumentales, como lectura y matemáticas.

Marchesi (2014) propone escuelas eficaces a través de la equidad y calidad educativa, términos inseparables. Para ello, es indispensable considerar los siguientes factores:

- Cuidado del contexto sociocultural de los alumnos;

- Un proyecto compartido por la comunidad educativa y con un liderazgo estable;

- Competencias y sensibilidad de los profesores para enseñar bien a todos sus alumnos y adaptarse a sus posibilidades;

- Orientación equilibrada hacia los objetivos de la educación: conocer, hacer, convivir y ser;

- Atención a los alumnos con dificultades de aprendizaje para reducir lo más posible las diferencias entre ellos;

- Apoyo especial a las escuelas que escolarizan a un mayor número de alumnos con dificultades para limitar las diferencias entre las diferentes escuelas;

- Disposición favorable hacia la innovación, y búsqueda de aliados y participación en redes educativas que contribuyan a alcanzar mejor estos objetivos. (p. 43)

Por consiguiente, es indispensable el desarrollo de políticas educativas inclusivas que debieran ser desarrolladas e implementadas con los fundamentos de acceso y calidad, igualdad y justicia social, democracia y participación, y equilibrio entre comunidad y diversidad, tal como lo sugiere Lázaro et al. (2015) en su propuesta descrita en la Tabla 3 factores para la mejora de la inclusión y cohesión social. 
TABLA 3

Factores para la mejora de la inclusión y cohesión social

\begin{tabular}{|c|c|c|c|}
\hline $\begin{array}{l}\text { 1. GESTIÓN } \\
\text { ESTRATÉGICA }\end{array}$ & $\begin{array}{l}\text { 2. GENERALIZACIÓN } \\
\text { DEL ACCESO A LA } \\
\text { TECNOLOGÍA }\end{array}$ & $\begin{array}{l}\text { 3. FORMACIÓN } \\
\text { PERMANENTE } \\
\text { DEL } \\
\text { PROFESORADO }\end{array}$ & $\begin{array}{l}\text { 4. EVALUACIÓN } \\
\text { DE POLÍTICAS } \\
\text { INCLUSIVAS }\end{array}$ \\
\hline $\begin{array}{l}\text { Políticas TIC a } \\
\text { medio y largo } \\
\text { plazo. } \\
\text { Continuidad } \\
\text { temporal. } \\
\text { Procesos de } \\
\text { diálogo. } \\
\text { Sostenibilidad } \\
\text { técnica y } \\
\text { financiera. } \\
\text { Planes } \\
\text { estratégicos. } \\
\text { Acuerdos entre } \\
\text { agentes sociales }\end{array}$ & $\begin{array}{l}\text { Atención NEE. Diseño } \\
\text { para todos. La } \\
\text { accesibilidad como } \\
\text { principio ético. } \\
\text { Equipamientos } \\
\text { públicos y gratuitos. }\end{array}$ & $\begin{array}{l}\text { Criterios de } \\
\text { accesibilidad. } \\
\text { Diseño } \\
\text { instruccional. } \\
\text { Tecnologias } \\
\text { emergentes. } \\
\text { Diseño para todos } \\
\text { en la formación } \\
\text { inicial. }\end{array}$ & $\begin{array}{l}\text { Identificación de } \\
\text { los factores de } \\
\text { éxito. Difusión de } \\
\text { trabajos. Datos } \\
\text { aislados. } \\
\text { Extrapolación o } \\
\text { transferencia } \\
\text { limitada. Bases de } \\
\text { datos } \\
\text { compartidas. }\end{array}$ \\
\hline
\end{tabular}

Fuente: Elaboración propia con datos de Lázaro et al. (2015).

Los centros educativos que favorecen la inclusión son aquellos los cuales muestran una organización institucional con enfoque cultural-pluralista y perspectiva de mejora escolar, es decir los que originen el cambio organizativo para generar procesos de "formación-innovación" (Moliner, 2013; p. 55) centrados en la escuela. Con lo anterior, Moliner (2013) menciona la existencia de varios modelos o instrumentos que facilitan la mejora y transformación de los centros escolares con orientación inclusiva; entre ellos se encuentra el index for inclusion o índice para la inclusión, por su traducción, que a continuación se describe.

Para tener una educación inclusiva, Booth y Ainscow (2000, citado por Moliner, 2013), proponen explorar la inclusión o exclusión a través del análisis de las situaciones que se presentan en la vida de la escuela considerando tres dimensiones interrelacionadas; estas a su vez presentan una serie de indicadores que son comprendidos como pretensiones para comparar la situación existente con la finalidad de establecer prioridades de desarrollo en la escuela, definidos en la Figura 4 dimensiones del índice de inclusión.

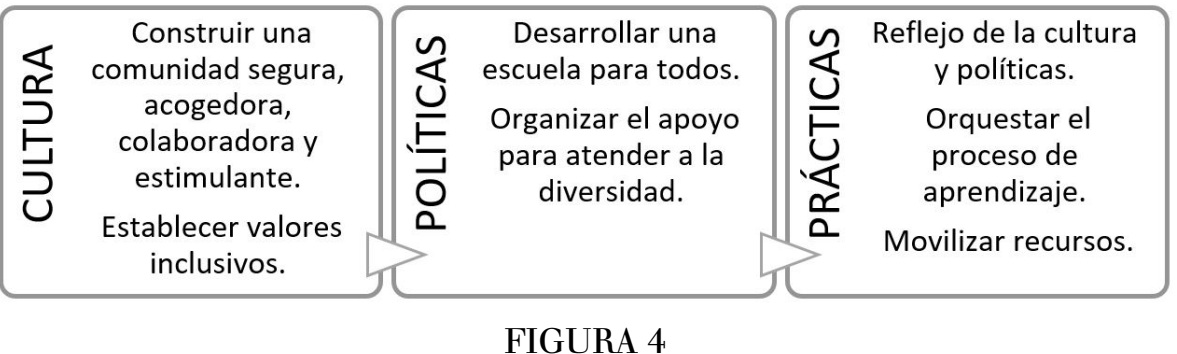

Dimensiones del índice para la inclusión

Fuente: Elaboración propia con datos de Moliner (2013)

Una vez que se tienen los resultados de la exploración se debe continuar con el proceso de mejora el cual, de acuerdo con Moliner (2013), está conformado por 5 etapas:

- Inicio del proceso del Índice.

- Análisis del centro.

- Elaboración de un plan de mejora con una orientación inclusiva

- Implementación de los aspectos susceptibles de mejora.

- Revisar el proceso del Índice (p.61). 
De acuerdo con el proceso del índice para la inclusión, se facilita la mejora de la inclusión mediante la participación de los integrantes de la comunidad, logrando llevar a cabo la transición del concepto de integración (solo participación del programa vigente) hacia la inclusión (transformación escolar hacia la diversidad del alumnado); con lo que, mediante lo indicadores obtenidos permite indagar y reconstruir colaborativamente el currículum (Moliner, 2013).

Los entornos inaccesibles generan discapacidad al crear barreras u obstáculos en la participación y la inclusión. Por ello se debe considerar la evaluación como un elemento importante en la toma de decisiones para logar la mejora continua y evitar esta dificultad.

\section{CONCLUSIONES}

Tras la revisión de 113 documentos publicados en la última década y el análisis exhaustivo de aproximadamente 50 de ellos que fueron considerados para la presente revisión bibliográfica, se obtuvieron las siguientes semblanzas.

La inclusión social es un reto que se presenta desde finales del siglo pasado, diversos organismos internacionales pretenden que sea superado en todos los países, mediante la reducción de la discriminación y marginación social en las personas más desfavorecidas por su situación personal, social, económica y/o cultural. No obstante, que cada uno de los países miembros de dichas organizaciones ha incorporado diversas políticas de inclusión propuestas en sus diferentes normativas, esto no ha sido suficiente por lo que se debe continuar los esfuerzos para avanzar en este sentido.

El ámbito educativo no está exento de la problemática anterior (Cabero, 2005). La educación inclusiva es una necesidad actual que debe ser incorporada en los diferentes sistemas educativos para favorecen la colaboración, los aprendizajes y el desarrollo de las potencialidades de cada uno de sus participantes, de tal manera, que esta contribuya significativamente en la conformación de sociedades más justas y equitativas.

Además, la educación inclusiva ha tratado de ampliar su conceptualización y no solo remitirse a padecimientos físicos, a pesar de los términos peyorativos con los cuales se asocia. Esta también considera las carencias económicas, sociales y culturales, que tornan vulnerables a los individuos que se encuentran desfavorecidos con el resto de la sociedad y el mismo sistema educativo. En adición a lo anterior, un modelo educativo de inclusión es un proceso de constante reflexión y reconstrucción, que por ende es una tarea compleja (Gallegos y López, 2018).

Las TIC en sus diversos usos y aplicaciones son una herramienta importante en el desarrollo de la educación inclusiva, que permiten hacer viables los principios de acceso, calidad, igualdad, justicia social, democracia, participación, buscando el equilibrio entre comunidad y diversidad. Por ende, las TIC al ser herramientas estratégicas consideradas dentro del aula para facilitar el aprendizaje formal de estudiantes, es necesario que docentes, diseñadores instruccionales y de materiales didácticos cuenten con el conocimiento, manejo y habilidades en TIC; pues con su destreza tecno-didáctica y tecno-pedagógica facilitan la democratización el conocimiento y promueven la equidad.

Es necesario advertir en algunos gobiernos latinoamericanos la falta de diferenciación entre los conceptos integración e inclusión, pues continúan manejándolos como sinónimos, siendo indispensable comprender su divergencia para la implementación del nuevo paradigma de inclusión educativa. A pesar del fuerte avance en los planes de incorporación de las TIC, en dichos países se observa una falta de políticas en la evaluación de su implementación, como en su seguimiento; por ende, muchos de estos programas van apareciendo y desapareciendo, intentando mejorar la calidad y potenciar la conectividad (Cabero y Valencia, 2019), enfatizando que todo ello se queda en papel o mientras dura el partido político en el gobierno. Complementando lo anterior, se debe concientizar la transformación de las prácticas educativas mediante las TIC, insistiendo en el magisterio con la formación del nuevo profesorado incidiendo en una especial 
atención del uso de las TIC para personas con diferentes tipos de discapacidades y para colectivos en riesgos de exclusión (Cabero y Valencia, 2019; Carrillo, y Molina, 2016).

Siendo indispensable recordar que las TIC permiten la potenciación de la producción de objetos de aprendizaje digitales que puedan ser utilizados abiertamente por el profesorado y el estudiantado, contextualizándolo en función de sus propias necesidades educativas. Considerando a los DU y DUA una herramienta tecnológica necesaria para lograr la inclusión educativa a través de los cuales auxilian a las personas que se encuentran en una situación vulnerable para la adquisición de conocimiento y desarrollo de sus aprendizajes. Inclusive Sánchez (2014) cita a autores tales como Ron Mace, et al. (2002), que proponen un modelo general sobre las características elementales, tales como invisibilidad, adaptabilidad y ubicuidad; y otros como, Rose y Meyer (2002) quienes sugieren que en los DUA las TIC funcionen como agentes activos dentro del aula.

En síntesis, las TIC son herramientas determinantes e indispensables para acercar democráticamente la educación a todas aquellas personas que se encuentran en situación de vulnerabilidad permitiendo su inclusión en la sociedad del siglo XXI. Para ello, sigue vigente la necesidad de elaborar, aplicar y evaluar políticas que aseguren la educación inclusiva, así como el acercamiento hacia una sociedad más equitativa y justa. Por consiguiente, se debe considerar la propuesta de cuatro factores centrales gestión estratégica, generalización de acceso a la tecnología, formación permanente del profesorado y evaluación de políticas inclusivas, además de la aplicación del modelo índice para la inclusión como instrumento evaluador y promotor de un currículo colaborativo.

En los años venideros debemos ser testigos no solo de nuevas políticas de inclusión educativa sino también de la evaluación de las ya existentes para así organizar una toma de decisiones a nivel local, nacional e internacional que promuevan una verdadera inclusión educativa con el apoyo de las tecnologías que se generen posteriormente.

\section{ReFERENCIAS}

Altamirano, S. y Lera, J, (2017). Futuro de las TIC's para una educación incluyente. En Jorge Lera (Organizador). II Congreso sobre Desigualdad Social, Económica y Educativa en el Siglo XXI. Llevado a cabo en el Grupo EUMEDUAT-UMA. Recuperado de https://bit.ly/338Ez4R

Baelo, R. y Cantón, I. (2009). Las tecnologías de la información y la comunicación en la educación superior. Estudio descriptivo y de revisión. Revista Iberoamericana de Educación, 50(7), 1-12. Recuperado de https://rieoei.org/ RIE/article/view/1965

Balladares, J. (2018). Competencias para una inclusión digital educativa. Revista PUCE. (107), 191-211. Recuperado de http://www.revistapuce.edu.ec/index.php/revpuce/article/view/179/261

Blanco, R. (2014). Inclusión educativa en América Latina: caminos recorridos y por recorrer. En A. Marchesi, R. Blanco, y L. Hernández, (Coords.). Avances y desafios en la educación inclusiva en Iberoamérica. (pp.11-35). Recuperado de https://www.oei.es/historico/noticias/spip.php?article13613

Cabero, J. (2005). Nuevas tecnologías y exclusión social un estudio sobre las posibilidades de las TIC en la lucha por la inclusión social en España. Madrid: Fundación Telefónica. Recuperado de https://bit.ly/2uJxhc4

Cabero, J. (2008). TICs para la igualdad: la brecha digital en la discapacidad. Anales, .8(2), 15-43. Recuperado de http s://dialnet.unirioja.es/servlet/articulo?codigo $=3622506$ (Error 1: El enlace externo https://dialnet unirioja.es/ servlet/articulo?codigo=3622506 debe ser una URL) (Error 2: La URL https://dialnet unirioja.es/servlet/ articulo?codigo $=3622506$ no esta bien escrita)

Cabero, J. (2010). Los retos de la integración de las TICs en los procesos educativos. Límites y posibilidades. Perspectiva Educacional, Formación de Profesores, 49(1), 32-61. Recuperado de https://www.redalyc.org/articulo.oa?id=33 3327288002 
Cabero, J. (2015). Inclusión digital-inclusión educativa. Sinergia, 2, 15-18. Recuperado de https://idus.us.es/bitstrea $\mathrm{m} /$ handle/11441/40763/SINERGIA_2Edici\%C3\%B3n.pdf?sequence=1\&isAllowed=y

Cabero, J. (2016). La educación a distancia como estrategia de inclusión social y educativa. Revista Mexicana de Bachillerato a Distancia, 8(15), 138-147. Recuperado de http://dx.doi.org/10.22201/cuaed.20074751e.2016. 15.57384

Cabero, J. y Córdoba, M. (2009). Inclusión educativa: inclusión digital. Revista de educación inclusiva, .2(1), 61-77. Recuperado de https://dialnetunirioja.es/servlet/articulo?codigo $=3011853$

Cabero, J. y Fernández, J. (2014). Una mirada sobre las tic y la educación inclusiva. Comunicación y pedagogía, (279-280), 38-42. Recuperado de https://bit.ly/2UQ0htl

Cabero, J., Fernández, J. y Córdoba, M. (2016). Conocimiento de las TIC aplicadas a las personas con discapacidades construcción de un instrumento de diagnóstico. Magis, 8(17), 157-176. doi: https://doi.org/10.11144/Javeria na.m8-17.ctap

Cabero, J. y Ruiz, P. (2018). Las Tecnologías de la información y la comunicación para la inclusión reformulando la brecha digital. International Journal of Educational Research and Innovation (IJERI), 9, 16-30. Recuperado de h ttps://www.upo.es/revistas/index.php/IJERI/article/view/2665/2222

Cabero, J. y Valencia, R. (2019). TIC para la inclusión una mirada desde Latinoamérica. Aula Abierta, 48(2), 39-146. doi: https://doi.org/10.17811/rifie.48.2.2019.139-146

Caridad, M., Calzada, F., Jorge, C., y Ayuso, M. (2011). Propuesta de un modelo para la medición del impacto en políticas TIC hacia la inclusión social: avances del proyecto IMPOLIS. Ciência da Informação, 40(2) 292-300. doi: https://doi.org/10.18225/ci.inf..v40i2.1317

Carrillo, M. y Molina, P. (2016). Inclusión social de las personas con discapacidad den las Américas. En B. Muñoz y A. Barrantes (Ed). Equidad e inclusión social: superando desigualdades hacia sociedades más inclusivas (OEA/Ser.D/ XXVI.15). (pp. 155-182). Washington: Organización de los Estados Americanos OEA. Recuperado de https: //bit.ly/3bClkpb

Castells, M. (2000). La sociedad red: La era de la información: economía, sociedad y cultura. Massachussets: Blackwell Publishers Inc. Recuperado de https://bit.ly/3bDb405

Consejo Nacional para el Desarrollo y la Inclusión de las Personas con Discapacidad [CONADIS]. (2015).Educación incluyente. [Mensaje de un blog]. CONADIS. Recuperado del https://www.gob.mx/conadis/es/articulos/edu cacion-incluyente?idiom $=\mathrm{es}$

Escobar, C., Restrepo, A. y Castrillón, A. (2016). Más allá de la brecha digital: la apropiación social de las tecnologías de la información y la comunicación (TIC) como opción para la inclusión. En B. Muñoz y A. Barrantes. (Ed). Equidad e inclusión social: superando desigualdades hacia sociedades más inclusivas (OEA/Ser.D/XXVI.15). (pp. 185-201). Washington: Organización de los Estados Americanos OEA. Recuperado de https://bit.ly/3bClkpb

Gallegos, T. y López, S. (2018). Inclusión y equidad en el nuevo modelo educativo. Revista de administración pública 145. 53(1) 77-86. Recuperado de http://www.inap.mx/portal/images/pdf/rap145.pdf

García, M. y López, R. (2012). Explorando, desde una perspectiva inclusiva, el uso de las tic para atender a la diversidad. Profesorado. Revista de Curriculum y Formación de Profesorado, 16(1), 277-293. Recuperado de https://www. redalyc.org/articulo.oa?id=56724377016

Gobierno de la República. (2013). Estrategia Digital Nacional. Recuperado de https://www.gob.mx/cms/uploads/at tachment/file/17083/Estrategia_Digital_Nacional.pdf

Lázaro, J., Estebanell, M. y Tedesco, J. (2015). Inclusión y cohesión social en una sociedad digital. RUSC. Universities and Knowledge Society Journal, 12(2), 44-59. doi: https://doi.org/10.7238/rusc.v12i2.2459

Levens, M. (2011). La desigualdad en la Educación en las Américas: Trabajando para crear oportunidades educativas para todos. En Organización de Estados Americanos. [OEA]. Desigualdad e inclusión social en las Américas: 14 ensayos. (OEA/Ser.D/XV.11) (pp. 191-213). Washington, D.C.: OEA. Recuperado de https://bit.ly/37pdBYf

Marchesi, A. (2014). Retos y dilemas de la inclusión educativa. En A. Marchesi, R. Blanco y L. Hernández. (Coord.). Avances y desafios de la educación inclusiva en Iberoamérica. España: Organización de Estados Iberoamericanos para la Educación, la Ciencia y la Cultura [OEI].. 
Moliner, O. (2013). Educación inclusiva. España: Universitat Jaume I. doi: http://doi.org/10.6035/Sapientia83

Organización de las Naciones Unidas para la Educación, la Ciencia y la Cultura [UNESCO]. (2009). Directrices sobre politicas de inclusión en la educación. París: UNESCO. Recuperado de https://bit.ly/31YV0Bh

Organización de las Naciones Unidas para la Educación, la Ciencia y la Cultura [UNESCO]. (2014). Conclusiones. En el Ministerio de Educación de España y OREALC/UNESCO Santiago. VIIIJornadas de Cooperación Educativa con Iberoamérica sobre Educación Especial e Inclusión Educativa. Llevado a cabo en UNESCO Montevideo, Uruguay. Recuperado de https://bit.ly/3bCFVtF

Organización Mundial de la Salud [OMS]. (2019). Temas de salud discapacidades. OMS. Recuperado de https://ww w.who.int/topics/disabilities/es/

Organización para la Cooperación y Desarrollo Económicos América Latina y el Caribe. [OCDE]. (2017). Mejorando la inclusión social en América Latina desafios clave y el rol de los sistemas de protección social OCDE. Recuperado https://bit.ly/3bG5MAK

Organización para la Cooperación y Desarrollo Económicos. [OCDE]. (2018). Perspectivas de la OCDE sobre la Economía Digital 2017. Ciudad de México: Asociación Mexicana de Internet doi: https://doi.org/10.1787/97 89264302211 -es.

Ortoll, E. (Coord.) (2007). La alfabetización digital en los procesos de inclusión social. Barcelona: UOC.

Palacios, J., Flores, E. y García, A. (2013). Diagnóstico del sector TIC en México Conectividad e inclusión social para la mejora de la productividad y el crecimiento económico. [s.l.]: Banco Interamericano de Desarrollo. Recuperado de https://bit.ly/37v7thc

Prioretti, J. (2016). TIC, TAC, TEP. Tecnologias para aprender y para toda la vida. Inclusión y calidad educativa. Recuperado de https://bit.ly/3bwvmZ0

Rodríguez, M. y Arroyo, M. (2014). Las TIC al servicio de la inclusión educativa. Digital Education Review, (25), 108-126. Recuperado de https://dialnetunirioja.es/servlet/articulo?codigo $=4778259$

Samaniego, P., Laitamo, S., Valerio, E. y Francisco, C. (2012). Informe sobre el uso de las tecnologias de información y comunicación (TIC) en la educación para personas con discapacidad. Quito: Organización de las Naciones Unidas para la Educación, la Ciencia y la Cultura (UNESCO). Recuperado de http://riberdis.cedd.net/handle/1118 $1 / 3589$

Sánchez, R. (2014). ¿Más avance tecnológico implica mayor inclusión? En el Ministerio de Educación de España y OREALC/UNESCO Santiago. VIII Jornadas de Cooperación Educativa con Iberoamérica sobre Educación Especial e Inclusión Educativa. Llevado a cabo en UNESCO Montevideo, Uruguay. Recuperado de https://bi t.ly/2tZ5jcb

Secretaría de Acceso y Equidad de Derechos [SADYE]. (2016). Garantizando más derechos para más gentes en las américas. Washington: OEA. Recuperado de http://www.oas.org/es/sadye/publicaciones/InformeGestion_SA DYE2016.pdf

Secretaría de Educación Pública. [SEP]. (2016). Programa @prende2.0 programa de inclusión digital 2016-2017.. México: SEP. Recuperado de https://bit.ly/2tZ6cBx

Souter, D. (2010). Towards inclusive knowledge societies a review of UNESCO's action in implementing the WSIS outcomes. France: UNESCO, Communication and Information Sector. Recuperado de https://unesdoc.unesc o.org/ark:/48223/pf0000187832

Thomas, H., Juárez, P. y Picabea, F. (2015). ¿Quéson las tecnologías de la inclusión social? Bernal: Universidad Nacional de Quilmes. Recuperado de https://issuu.com/redtisa/docs/cuadernillo_n1_online

Vaillant, D. (2013). Las politicas TIC en los sistemas educativos de América Latina: caso Uruguay. Argentina: UNICEF. Recuperado de https://bit.ly/2HnjtqH

Velasco, L. (2017). El proceso de inclusión de las personas con discapacidad desde el plan nacional de desarrollo 2013-2018. Su implementación en el caso de Jalisco, México. Revista inclusiones, 4(Especial), 102-117. Recuperado de http://repositoriocdpd.net:8080/handle/123456789/2006 
Rafael Reyes Chávez, et al. Las Tecnologías de Información y Comunicación como herramienta para un...

Watkins, A. (Ed.). (2013). Tecnologias de la información y la comunicación para la inclusión Avances y oportunidades en los paises europeos. Bélgica: Agencia Europea para el Desarrollo de la Educación del Alumnado con Necesidades Educativas Especiales. Recuperado de https://bit.ly/39xBHBI

Watts, C.y Lee, L. (2017). Las TIC como herramientas de inclusión educativa. Acta Scientiae Informaticae, 1(1), 92-97. Recuperado de https://bit.ly/2OTWasy

Zappalá, D., Köppel, A. y Suchodolski, M. (2011). Inclusión de tic en escuelas para alumnos con discapacidad intelectual. Buenos Aires: Ministerio de Educación de la Nación. Recuperado de https://bit.ly/2OVm5zZ

\section{BY-NC-ND}

